\title{
Comparison of the Long-Range Climate Memory in Outgoing Longwave Radiation over the Tibetan Plateau and the Indian Monsoon Region
}

\author{
Zhongping Shen, ${ }^{1}$ Jun Shi, ${ }^{1}$ and Yadong Lei $^{2}$ \\ ${ }^{1}$ Shanghai Climate Center, Shanghai, China \\ ${ }^{2}$ Key Laboratory of Meteorological Disaster, Ministry of Education, Nanjing University of Information Science and Technology, \\ Nanjing, China \\ Correspondence should be addressed to Zhongping Shen; shenzhongping126@126.com
}

Received 26 May 2017; Revised 27 July 2017; Accepted 23 August 2017; Published 28 September 2017

Academic Editor: Jia Yue

Copyright (C) 2017 Zhongping Shen et al. This is an open access article distributed under the Creative Commons Attribution License, which permits unrestricted use, distribution, and reproduction in any medium, provided the original work is properly cited.

Based on the detrended fluctuation analysis (DFA) method, scaling behaviors of the daily outgoing longwave radiation (OLR) from 1979 to 2015 over the Tibetan Plateau (TP) and the Indian Monsoon Region (IMR) are analyzed. The results show that there is long-term memory for the OLR time series over the TP and IMR. The long-range memory behaviors of OLR over TP are stronger than those over IMR. The averaged values of the scaling exponents over TP and IMR are 0.71 and 0.64 ; the maximum values in the two regions are 0.81 and 0.75 ; the minimum values are 0.59 and 0.58 . The maximum frequency counts for scaling exponents occur in the range of 0.625 and 0.675 both in TP and in IMR. The spatial distribution of the scaling exponents of the OLR sequence is closely related to the conditions of climatic high cloud cover in the two areas. The high cloud cover over TP is obviously less than that of IMR. In addition, the scaling behaviors of OLR over TP and IMR are caused by the fractal characteristics of time series, which is further proved by randomly disrupting the time series to remove trends and correlation.

\section{Introduction}

As is known to all, the change of a climate system has the selfmemory characteristic. This means that the past climate has a long-term effect on the variational trend of the current and future climate system. Long-range memory (LRM), which is also called the long-range correlation (LRC) or long-term persistence, has been found in many observations, such as daily temperature records [1-6], daily precipitation records $[7,8]$, relative homogeneity $[9,10]$, sea surface temperature [11], ground surface temperature $[8,12]$, and geopotential height [13]. LRM is characterized by a diverging integral time scale and linked to the power-law behavior of the autocorrelation function [5]. In a complex climate system, a copious amount of signals is nonstationary, whose mean, standard deviation, and autocorrelation function change with time [14-17]. Traditional methods such as the power spectrum analysis or the correlation analysis are suitable for calculating relevant characteristics of stationary signals. In order to reliably analyze the long-range correlation of time series, it is necessary to identify the trend components generated by the inherent long-range fluctuation. If they are not filtered out, in the long-range correlation analysis, there will be spurious information caused by strong trend components. The detrended fluctuation analysis (DFA) method is powerfully used to reveal the extent of long-range correlations in time series $[18,19]$. It can filter out the trend variation first and then disclose the persistence characteristics of a time series. At present, DFA has been widely used to analyze the LRMs in many types of time series, especially for the basic meteorological elements such as daily temperature and precipitation. Recently, the scaling behaviors of outgoing longwave radiation (OLR) records in the tropic are discussed by Lei et al. [20]. However, fewer efforts have been dedicated to studying the scaling behaviors of OLR records over the TP and IMR. 
OLR is the energy radiating from the earth to external space as infrared radiation. It has a close relationship with earth-atmosphere radiation budget $[21,22]$. It mainly depends on the temperature profile (the cloud in the cloudy sky or the underlying surfaces in the clear sky) [23, 24]. In view of relative homogeneity of the underlying surfaces in low-latitude and midlatitude zones, the value of OLR is mainly determined by the cloud, such as cloud top temperature and cloudiness. The cloud top temperature hinges on the strength of convection. The stronger the convection is, the lower the cloud top temperature is. Therefore, OLR can reveal the intensity of convective activity to some extent. There is a close inverse correlation between OLR and precipitation in tropical and subtropical areas. Precipitation of TP is mainly affected by the Indian monsoon, while the heating of TP has a strong effect on precipitation of IMR. In addition, the domains of TP and IMR show different climatic characteristics. Therefore, the research on scaling behaviors of OLR over TP and IMR is helpful for us to understand the climate dynamics. Also, the long-range correlation of the OLR provides theoretical support for precipitation prediction.

In this study, we investigate the long-range correlation of the OLR from 1979 to 2015 over the Tibetan Plateau (TP) and the Indian Monsoon Region (IMR) and compare the differences between them. The results indicate that the variation of OLR is not random but demonstrate obvious LRC. This paper is organized as follows. In Section 2, the details of the OLR time series are provided, and the DFA method is briefly described. In Section 3, we detect the LRC and analyze the spatial distributions of scaling exponents in OLR. The discussions and conclusions follow in Section 4.

\section{Datasets and Method}

2.1. Datasets. The daily OLR time series between 1979 and 2015 are downloaded from the website of the National Oceanic and Atmospheric Administration (https://www.esrl .noaa.gov/psd/data/gridded/data.interp_OLR.html). The spatial resolution of this dataset is $2.5^{\circ} \times 2.5^{\circ}$ [25]. The cloud data is obtained from the European Centre for Medium-Range Weather Forecasts (http://apps.ecmwf.int/datasets/data/interimfull-moda/levtype=sfc/).

2.2. Description of the DFA Method. The generalized DFA method, which was introduced by Peng et al. [19], is a powerful technique to study LRCs in time series. It has been widely accepted in the past years because of its capability to tackle nonstationary signals $[7,26]$. The calculation steps of DFA are described below.

We first remove the annual cycle from the raw data $R_{i}$ (daily mean OLR) by computing the anomaly series as follows:

$$
x_{i}=R_{i}-\left\langle R_{i}\right\rangle_{d},
$$

where $\left\langle R_{i}\right\rangle_{d}$ represents the climatological mean for the given calendar day $d=1,2, \ldots, 366$ (when leap days are included).
Then, we determine the cumulative deviation series as follows:

$$
y(j)=\sum_{i=1}^{j} \Delta x_{i}, \quad(j=1,2,3, \ldots, N),
$$

where $\Delta x_{i}=x_{i}-\bar{x}$ and $\bar{x}$ denotes the mean value of the series $x_{i}(i=1,2,3, \ldots, N)$.

Next, we divide the series $y(j)$ into $m$ nonoverlapping segments with equal length $n$, where $m$ is the integer part of $N / n$. In order to make full use of the remaining data left over from the tail, the series $y(j)$ is redivided from back to front. Consequently, we get $2 m$ segments. For a p-order DFA process, a p-order polynomial is used to calculate the local trend in each segment. A second-order DFA (DFA2) method, which is the most commonly used order, is employed in this work. In each segment $v$, we apply a least-squares fit to get the local trend $\tilde{y}(j, v)$ and the trends of the profile are removed by subtracting the local fits. The variance is determined as follows:

$$
\begin{aligned}
& F^{2}(n, v) \\
& = \begin{cases}\frac{1}{n} \sum_{j=1}^{n}(y(j, v)-\tilde{y}(j, v))^{2}, & (v=1,2, \ldots, m) \\
\frac{1}{n} \sum_{j=1}^{n}(y(j, v)-\tilde{y}(j, v))^{2}, & (v=m+1, m+2, \ldots, 2 m) .\end{cases}
\end{aligned}
$$

The detrended fluctuation function is obtained with the arithmetic mean of the variance in all segments:

$$
F(n)=\sqrt{\frac{1}{2 m} \sum_{v=1}^{2 m}\left(F^{2}(n, v)\right) .}
$$

If the fluctuation function $F(n)$ increases with window length $n$ in accordance with the power-law relation, $F(n) \sim$ $n^{\alpha}$. When the scaling exponent $\alpha>0.5$, then the time series show positive long-term correction. If the scaling exponent $\alpha<0.5$, then the time series show long-term negative longterm correlation. For scaling exponent $\alpha=0.5$, the time series is random.

\section{Result}

Two grid points, $\left(95^{\circ} \mathrm{E}, 35^{\circ} \mathrm{N}\right)$ and $\left(97.5^{\circ} \mathrm{E}, 12.5^{\circ} \mathrm{N}\right)$, located in TP and IMR, respectively, are chosen randomly to analyze the scaling behaviors of OLR time series. The temporal evolution of OLR anomaly and cumulative deviation during the period from 1979 to 2015 is shown in Figure 1. It is found that both of the OLR anomaly records present irregular highfrequency fluctuations in Figures 1(a) and 1(c). The original cumulative deviation series are shown in Figures 1(b) and 1(d) with black lines, and the shuffled cumulative deviation series are shown with red lines. For the grid point $\left(95^{\circ} \mathrm{E}, 35^{\circ} \mathrm{N}\right)$ of TP (Figure 1(b)), the original cumulative deviation series of OLR deceases between 1979 and 2003 and then increases after 2003. The minimum value appears around 2003. For the grid point $\left(97.5^{\circ} \mathrm{E}, 12.5^{\circ} \mathrm{N}\right)$ of IMR (Figure $1(\mathrm{~d})$ ), the original cumulative deviation series of OLR has a strong peak. The 


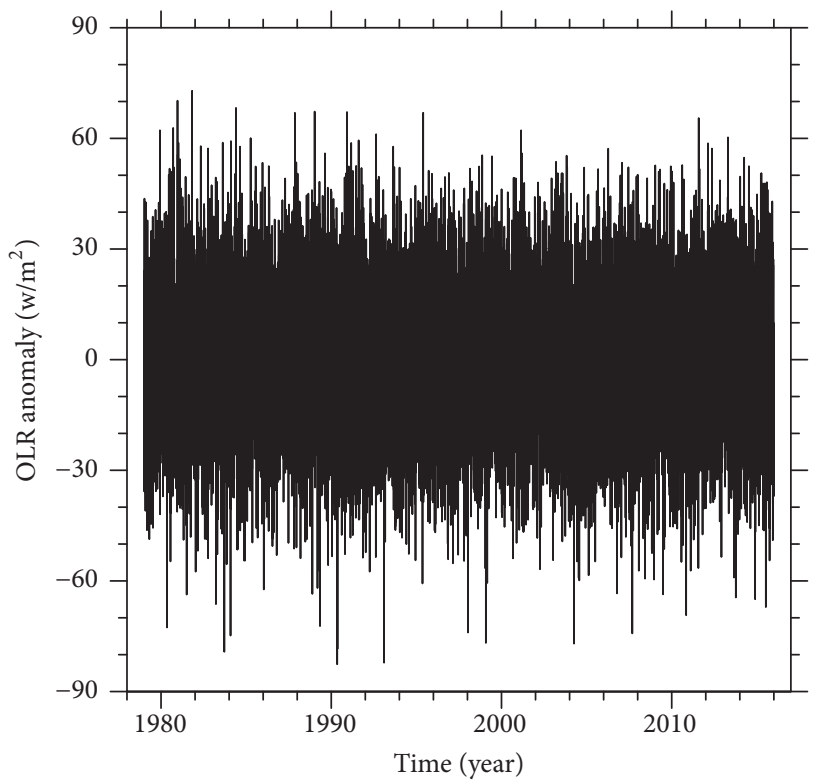

(a)

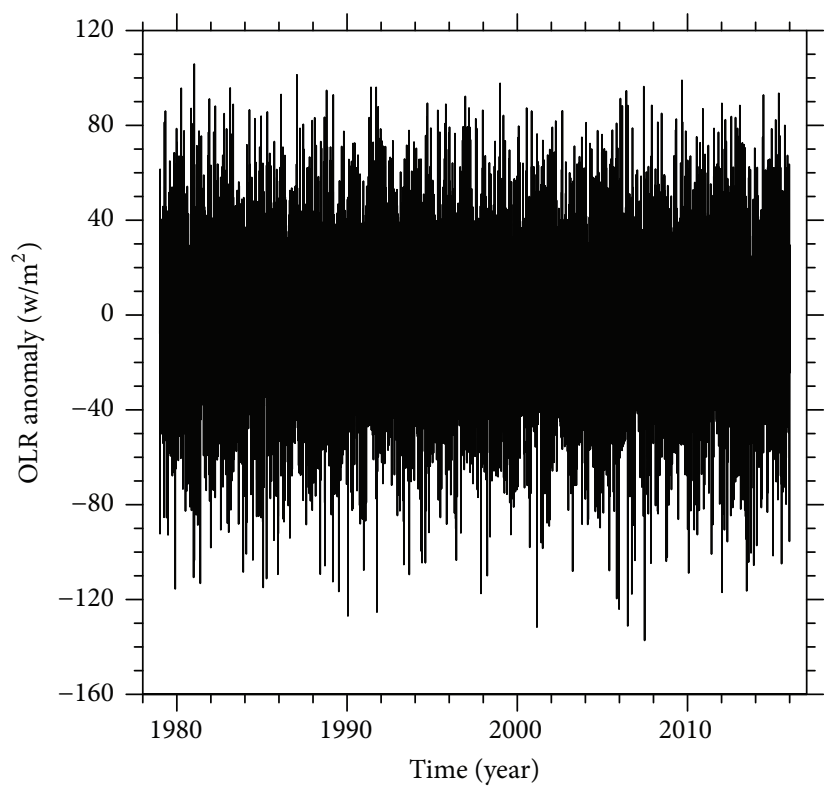

(c)

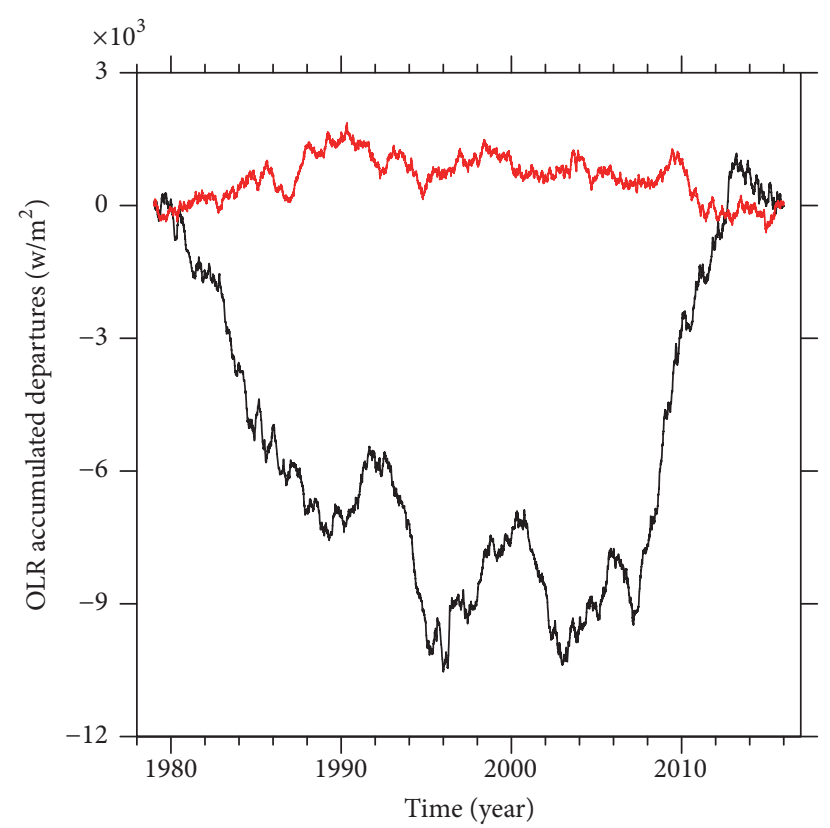

(b)

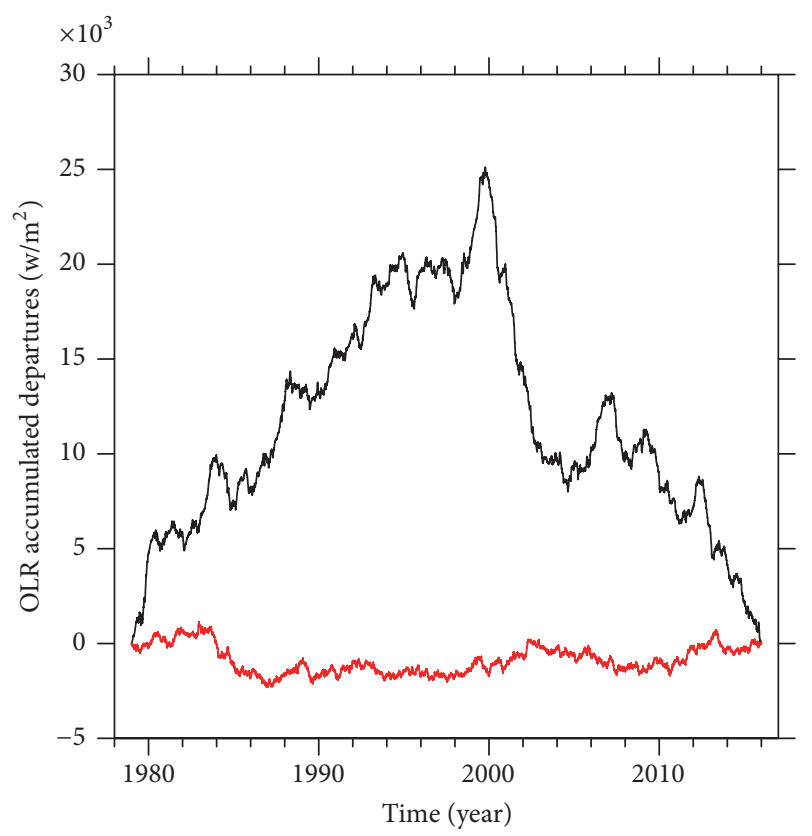

(d)

FIGURE 1: The anomaly and cumulative deviation of OLR in TP (a, b) and IMR (c, d). The black and red line in (b) and (d) represent the original and shuffled time series, respectively.

maximum value occurs around 1998. There are remarkable differences between the two grid points. For the randomly shuffled cumulative deviation series of OLR, there are no significant fluctuations in the two grid points, which means that relevance in the times series is destroyed by the random shuffle. In order to investigate the scaling behaviors of OLR time series, different orders of DFA are used in the following discussion. DFA1-DFA4 are used in the following discussion. The double log plots of power-law relationship between the detrended variability $F(n)$ and the window scale $n$ for the two grid points are presented in Figure 2. It is shown that the slope is approximately linear for the four different orders of DFA, and therefore there are obviously similar scaling behaviors by using the different orders of DFA for the two grid points. DFA1 can only eliminate the linear trends, while DFA2-DFA4 can be used to remove the nonlinear trends in the time series. In addition, the slope of DFA2 is the most linear among DFA2-DFA4. Therefore, DFA2, which is able to remove unexpected trends, is adopted to analyze LRC of OLR. The black solid line represents the linear fits of OLR 


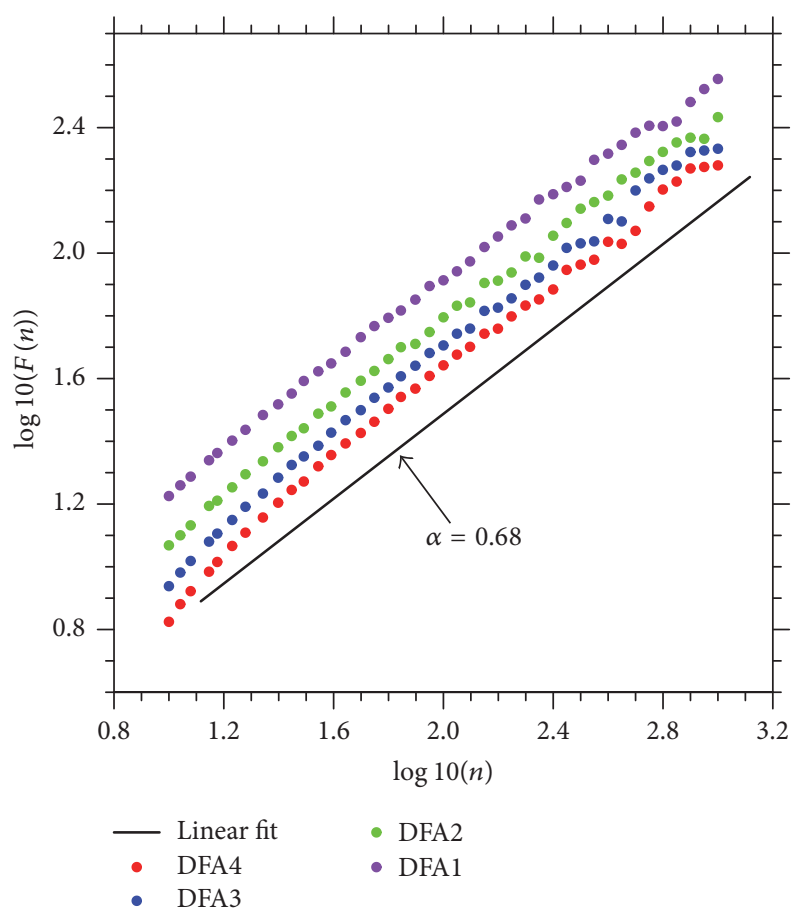

(a)

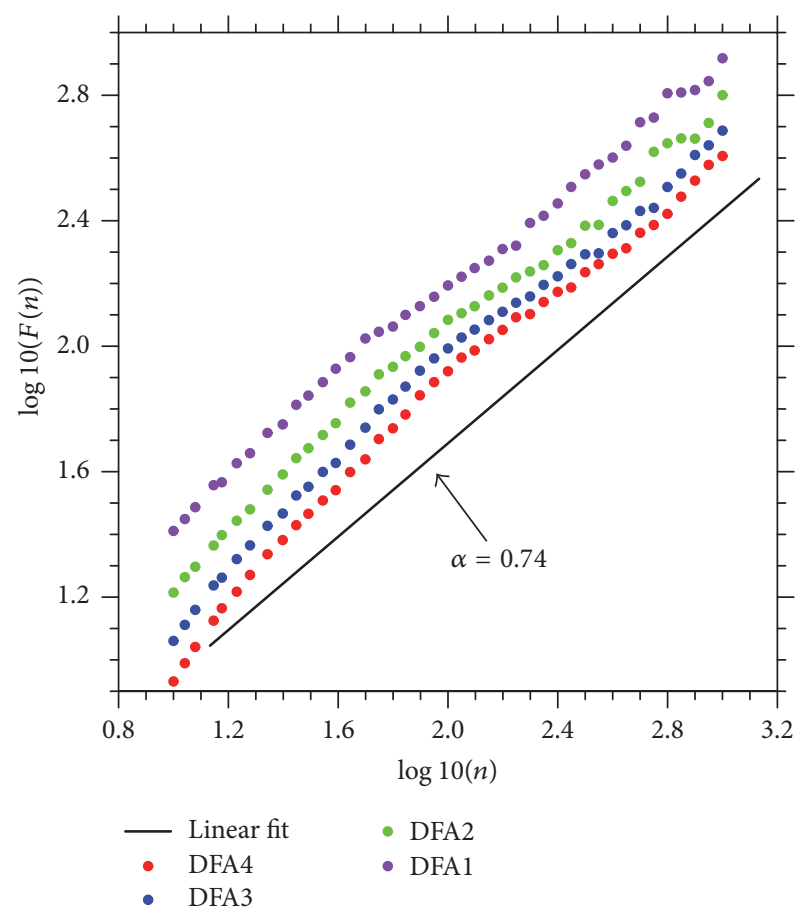

(b)

Figure 2: The double log plots of power-law relationship between the detrended variability $F(n)$ and the time scale $n$ for the two grid points $\left(95^{\circ} \mathrm{E}, 35^{\circ} \mathrm{N}\right)$ and $\left(97.5^{\circ} \mathrm{E}, 12.5^{\circ} \mathrm{N}\right)$ using DFA1-DFA4. The black solid line represents linear fit. (a) is for the grid point $\left(95^{\circ} \mathrm{E}, 35^{\circ} \mathrm{N}\right)$ and $(\mathrm{b})$ is for the grid point $\left(97.5^{\circ} \mathrm{E}, 12.5^{\circ} \mathrm{N}\right)$.

sequence at the two grid points. The scaling exponent $\alpha$ at the grid points $\left(95^{\circ} \mathrm{E}, 35^{\circ} \mathrm{N}\right)$ and $\left(97.5^{\circ} \mathrm{E}, 12.5^{\circ} \mathrm{N}\right)$ is equal to 0.68 and 0.74 , respectively. This means that there exist positive long-range correlation behaviors. Figure 3 illustrates the double log plots of the power-law relationship between the detrended variability $F(n)$ and the window scale $n$ of the random shuffle (randomly disrupting the daily OLR time series). It shows that scaling exponent $\alpha$ is close to 0.5 , thus demonstrating white noise characteristics, which verifies that the long-term persistence of OLR sequence is caused by the fractal characteristics of the sequence itself.

In order to illustrate the LRC of the OLR sequence over TP and IMR in an overall manner, the spatial distribution of the scaling exponents is shown in Figure 4. It can be seen that the scaling exponents of OLR present a decreasing trend from west to east over TP. Among them, the scaling index of OLR over the southwestern TP is as high as 0.76 , indicating strong long-term memory characteristic, while that of the northeastern TP is less than 0.64 , exhibiting weak long-term memory characteristic. For IMR, it is presented that the scaling exponents of OLR reach the minimum value of less than 0.58 in the Indian Ocean. In the land part of IMR, the scaling exponents of OLR can reach as high as 0.66 , even 0.70 in the northwest. The above distribution features are closely related to the conditions of climatic cloud cover in the two regions. In a cloudy sky, OLR usually depends on the highest cloud top temperature and cloudiness. The variation of cloud top temperature is quick for its reliance on the generation, development, and extinction of the cloud. Therefore, in the cloudy sky, the LRC of OLR is weak. However, OLR mainly depends on the brightness temperature of underlying surfaces in clear sky and it changes slowly, which causes the LRC of OLR to be strong. Figure 5 shows the spatial distribution of high cloud over TP and IMR. The high cloud cover over TP is obviously less than that of IMR, which is the reason why the LRC of the OLR sequence over TP is stronger than that of IMR. For the TP region, the cover of cloud increases from west to east, which makes the LRM decrease from west to east over TP. In the Indian Ocean region, there exists a strong convection, which leads to larger high cloud cover. Besides, the LCR of OLR is also affected by other factors such as the atmosphere temperature profile and relative humidity. Therefore, the spatial distribution of scaling exponents is not strictly consistent with that of the cloud cover.

In order to further describe the differences between the two regions, the frequency distribution of the scaling exponents of OLR is given in Figure 6 and the statistical characteristics are illustrated in Table 1 . The maximum frequency counts of scaling exponents occur in the range of 0.67 and 0.69 over both TP and IMR. In Table 1, the averaged values of the scaling exponents of OLR over TP and IMR are 0.71 and 0.64 , respectively; the maximum values of the scaling exponents of OLR in the two regions are 0.81 and 0.75 , respectively; the minimum values of the scaling exponents of OLR in the two regions are 0.59 and 0.58 , respectively. In general, there is a stronger long-term memory characteristic over TP than that over IMR. The mean value of shuffled OLR records is 0.50 for the two regions. The minimum values of 


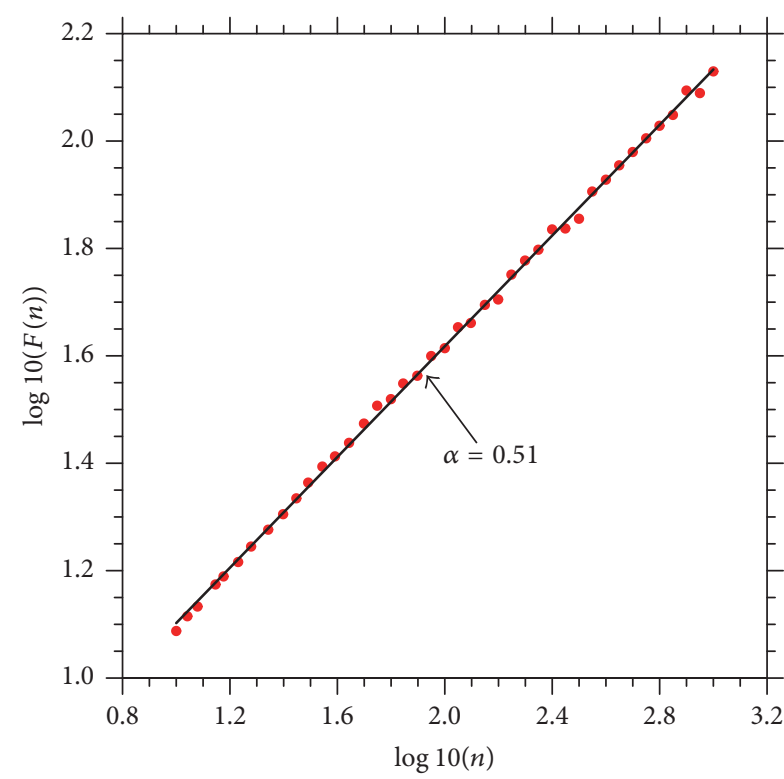

(a)

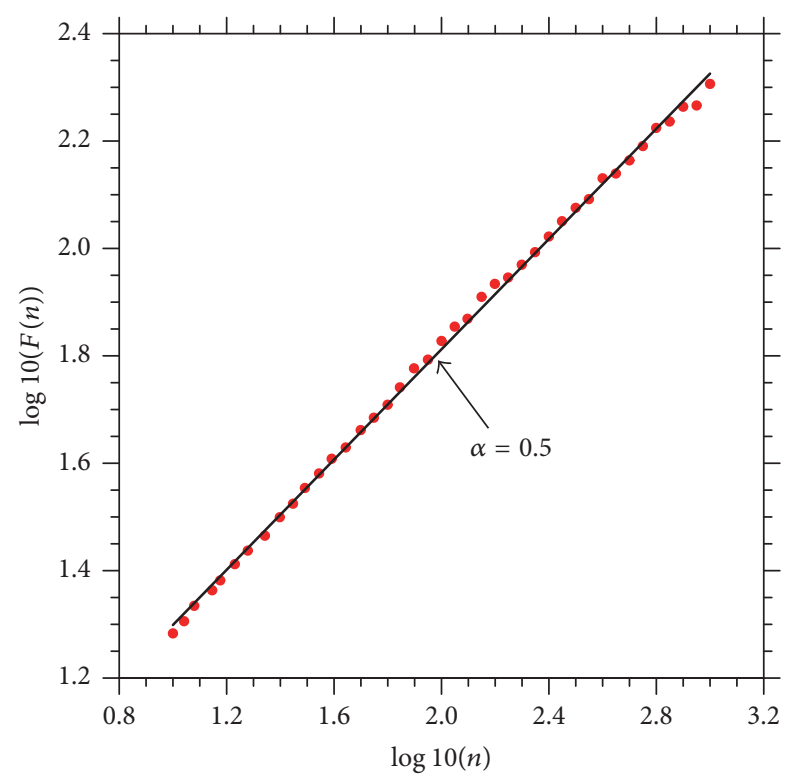

(b)

FIGURE 3: The double log plots of the power-law relationship between the detrended variability $F(n)$ and the time scale $n$ for randomly shuffled OLR of the two grid points $\left(95^{\circ} \mathrm{E}, 35^{\circ} \mathrm{N}\right)$ and $\left(97.5^{\circ} \mathrm{E}, 12.5^{\circ} \mathrm{N}\right)$. The black solid lines are linear fit. (a) is for the grid point $\left(95^{\circ} \mathrm{E}, 35^{\circ} \mathrm{N}\right)$ and $(\mathrm{b})$ is for the grid point $\left(97.5^{\circ} \mathrm{E}, 12.5^{\circ} \mathrm{N}\right)$.

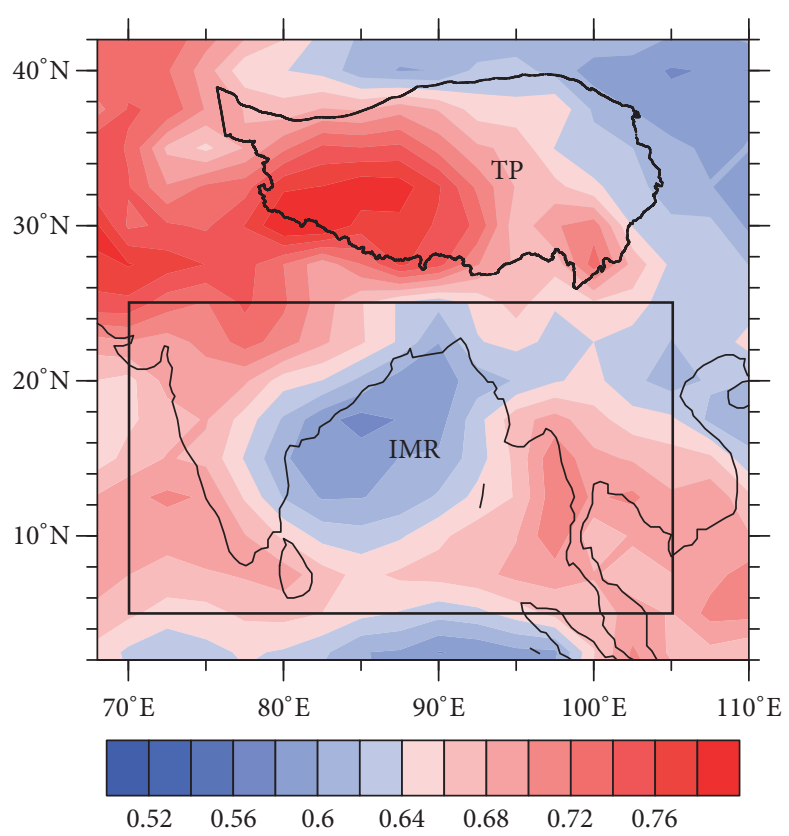

FIGURE 4: The geographical distribution of the scaling exponents of the daily OLR sequence over TP and IMR.

TABLE 1: The main parameters of scaling exponents of the OLR sequence on TP and IMR.

\begin{tabular}{lcccc}
\hline DFA2 & Minimum & Maximum & Average & Standard deviation \\
\hline TP & 0.59 & 0.81 & 0.71 & 0.042 \\
IMR & 0.58 & 0.75 & 0.64 & 0.036 \\
Shuffled_TP & 0.46 & 0.58 & 0.50 & 0.016 \\
Shuffled_IMR & 0.48 & 0.56 & 0.50 & 0.012 \\
\hline
\end{tabular}




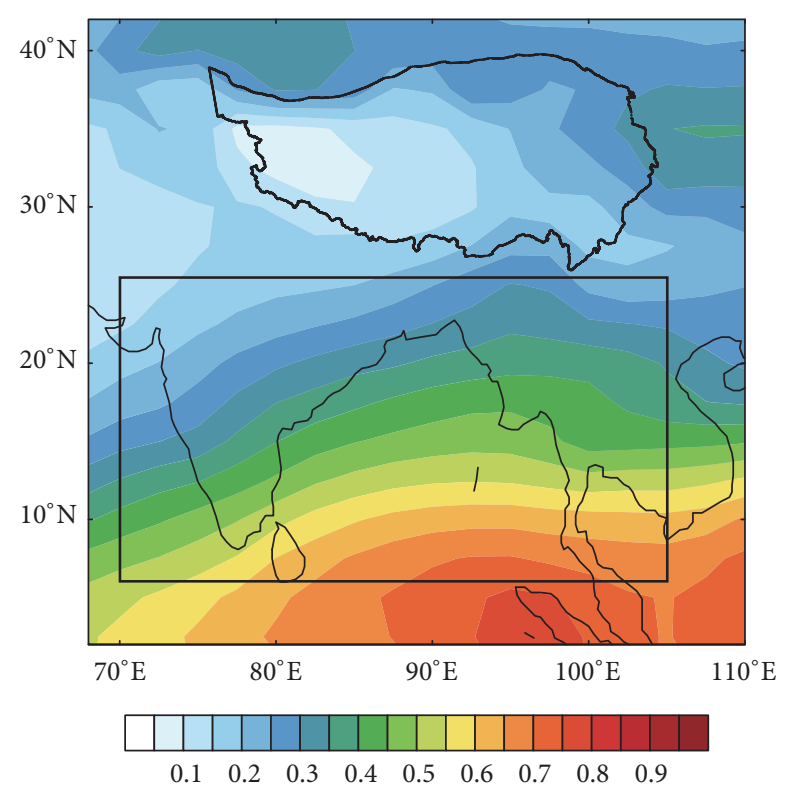

FIGURE 5: The spatial distribution of total cloud over TP and IMR.

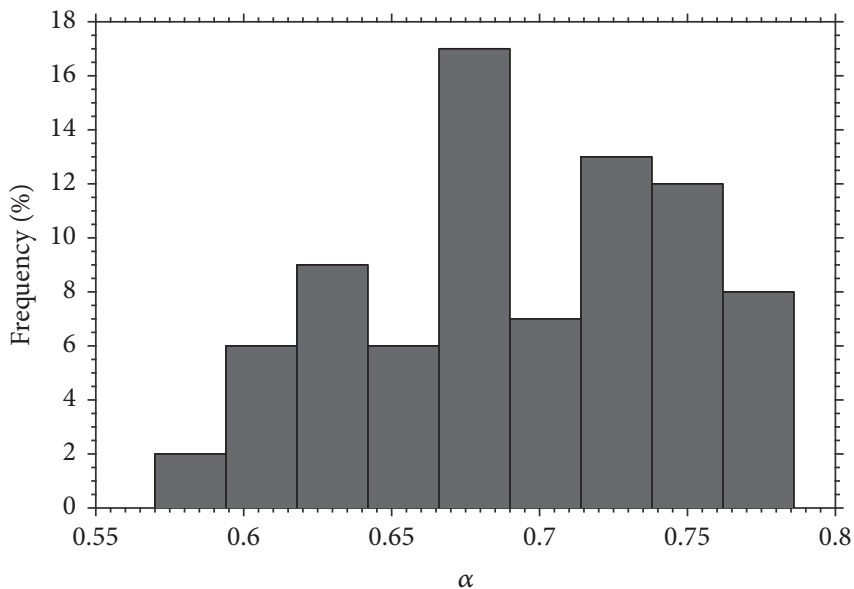

(a)

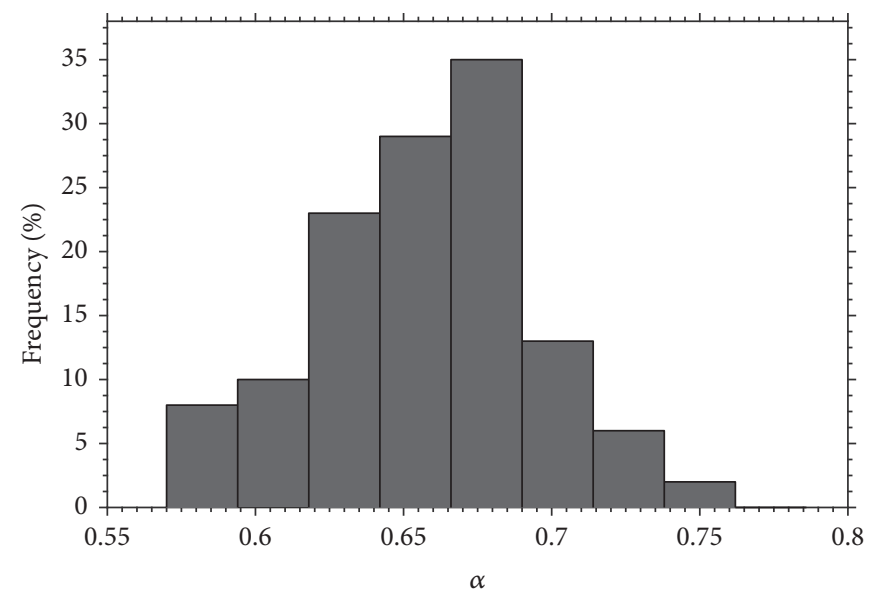

(b)

FIgURE 6: The frequency distribution of the scaling exponents of OLR over TP (a) and IMR (b).

shuffled OLR records in the two regions are very close, and so are their maximum values. In other words, there are no observable differences between shuffled OLR records in the two places, which further proves that LRC behaviors of OLR in the two regions are caused by the fractal characteristics of time series.

\section{Conclusion and Discussion}

In this paper, the DFA method is used to analyze the longterm memory characteristic over TP and IMR. The two grid points, $\left(95^{\circ} \mathrm{E}, 35^{\circ} \mathrm{N}\right)$ and $\left(97.5^{\circ} \mathrm{E}, 12.5^{\circ} \mathrm{N}\right)$, located in $\mathrm{TP}$ and IMR, respectively, are chosen randomly to analyze the scaling behaviors of OLR time series by using the different orders of DFA. The results present the notion that the slope is approximately linear for the four different orders of
DFA, which means that there exist obviously similar scaling behaviors by using the different orders of DFA. Among them, DFA2 can remove unexpected trends, and therefore it is used to calculate LRC over the two regions. Generally, the scaling exponents in IMR are smaller than those of TP, which indicates stronger long-term memory characteristic for TP. Meanwhile, the scaling exponents of OLR have a decreasing trend from west to east over TP. The maximum frequency counts of scaling exponents occur in the range of 0.67 and 0.69 both in TP and in IMR. The spatial distribution feature of the scaling exponents of the OLR sequence in the two regions is mainly affected by that of the cloud cover. The high cloud cover over TP is obviously less than that of IMR. For TP region, the cover of cloud increases from west to east, and consequently the LRM becomes weaker from west to east over TP. In the Indian Ocean region, there exists a strong 
convection, which leads to larger high cloud cover. The scaling exponents of shuffled OLR records in the two places are near 0.5 , which illustrates that LRC behaviors of OLR in the two regions are caused by the fractal characteristics of time series.

\section{Conflicts of Interest}

The authors declare that they have no conflicts of interest.

\section{Acknowledgments}

This study was funded by the National Natural Science Foundation of China (nos. 41571044 and 41001283) and by the Climate Change Special Fund of the China Meteorological Administration (no. CCSF201716).

\section{References}

[1] E. Koscielny-Bunde, A. Bunde, S. Havlin, and Y. Goldreich, "Analysis of daily temperature fluctuations," Physica A Statistical Mechanics \& Its Applications, pp. 231-393, 1996.

[2] P. Talkner and R. O. Weber, "Power spectrum and detrended fluctuation analysis: application to daily temperatures," Physical Review E, vol. 62, no. 1, 150 pages, 2000.

[3] R. O. Weber and P. Talkner, "Spectra and correlations of climate data from days to decades," Journal of Geophysical Research Atmospheres, vol. 106, no. D17, pp. 20131-20144, 2001.

[4] A. Bunde and S. Havlin, "Power-law persistence in the atmosphere and in the oceans," Physica A: Statistical Mechanics and its Applications, vol. 314, no. 1-4, pp. 15-24, 2002.

[5] K. Fraedrich and R. Blender, "Scaling of atmosphere and ocean temperature correlations in observations and climate models," Physical Review Letters, vol. 90, Article ID 108501, 2003.

[6] J. F. Eichner, E. Koscielny-Bunde, A. Bunde, S. Havlin, and H.-J. Schellnhuber, "Power-law persistence and trends in the atmosphere: A detailed study of long temperature records," Physical Review E, vol. 68, Article ID 046133, 2003.

[7] J. W. Kantelhardt, E. Koscielny-Bunde, D. Rybski, P. Braun, A. Bunde, and S. Havlin, "Long-term persistence and multifractality of precipitation and river runoff records," Journal of Geophysical Research Atmospheres, vol. 111, no. 1, Article ID D01106, 2006.

[8] L. Jiang, N. Li, and X. Zhao, "Scaling behaviors of precipitation over China," Theoretical and Applied Climatology, vol. 128, no. 1-2, pp. 63-70, 2017.

[9] X. Chen, G. Lin, and Z. Fu, "Long-range correlations in daily relative humidity fluctuations: A new index to characterize the climate regions over China," Geophysical Research Letters, vol. 34, no. 7, Article ID L07804, 2007.

[10] G. Lin, X. Chen, and Z. Fu, "Temporal-spatial diversities of long-range correlation for relative humidity over China," Physica A: Statistical Mechanics and its Applications, vol. 383, no. 2, pp. 585-594, 2007.

[11] M. Luo, Y. Leung, Y. Zhou, and W. Zhang, "Scaling behaviors of global sea surface temperature," Journal of Climate, vol. 28, no. 8, pp. 3122-3132, 2015.

[12] L. Jiang, N. Li, Z. Fu, and J. Zhang, "Long-range correlation behaviors for the $\mathrm{o}-\mathrm{cm}$ average ground surface temperature and average air temperature over China," Theoretical and Applied Climatology, vol. 119, no. 1-2, pp. 25-31, 2015.

[13] A. A. Tsonis, P. J. Roebber, and J. B. Elsner, "Long-range correlations in the extratropical atmospheric circulation: Origins and implications," Journal of Climate, vol. 12, no. 5, pp. 1534-1541, 1999.

[14] M. C. Casdagli, "Recurrence plots revisited," Physica D. Nonlinear Phenomena, vol. 108, no. 1-2, pp. 12-44, 1997.

[15] P. F. Verdes, P. M. Granitto, H. D. Navone, and H. A. Ceccatto, "Nonstationary time-series analysis: Accurate reconstruction of driving forces," Physical Review Letters, vol. 87, no. 12, Article ID 124101, 2001.

[16] P. Yang, G. Wang, F. Zhang, and X. Zhou, "Causality of global warming seen from observations: a scale analysis of driving force of the surface air temperature time series in the Northern Hemisphere," Climate Dynamics, vol. 46, no. 9-10, pp. 31973204, 2016.

[17] F. Zhang, P. Yang, K. Fraedrich, X. Zhou, G. Wang, and J. $\mathrm{Li}$, "Reconstruction of driving forces from nonstationary time series including stationary regions and application to climate change," Physica A. Statistical Mechanics and its Applications, vol. 473, pp. 3197-3204, 2017a.

[18] C.-K. Peng, J. Mietus, J. M. Hausdorff, S. Havlin, H. E. Stanley, and A. L. Goldberger, "Long-range anticorrelations and nonGaussian behavior of the heartbeat," Physical Review Letters, vol. 70, no. 9, pp. 1343-1346, 1993.

[19] C.-K. Peng, S. V. Buldyrev, S. Havlin, M. Simons, H. E. Stanley, and A. L. Goldberger, "Mosaic organization of DNA nucleotides," Physical Review E, vol. 49, no. 2, pp. 1685-1689, 1994.

[20] Y. Lei, F. Zhang, Q. Yang, J. Zhang, L. Jiang, and R. Liu, "Longterm memory behaviors for outgoing longwave radiation in the tropics," Journal of Tropical Meteorology, vol. 33, no. 3, pp. 426432, 2017.

[21] K. Wu, F. Zhang, J. Min, Q.-R. Yu, X.-Y. Wang, and L. $\mathrm{Ma}$, "Adding method of delta-four-stream spherical harmonic expansion approximation for infrared radiative transfer parameterization," Infrared Physics and Technology, vol. 78, pp. 254262, 2016.

[22] F. Zhang, K. Wu, J. Li, Q. Yang, J.-Q. Zhao, and J. Li, “Analytical infrared delta-four-stream adding method from invariance principle," Journal of the Atmospheric Sciences, vol. 73, no. 10, pp. 4171-4188, 2016.

[23] F. Zhang, K. Wu, P. Liu, X. Jing, and J. Li, "Accounting for Gaussian quadrature in four-stream radiative transfer algorithms," Journal of Quantitative Spectroscopy and Radiative Transfer, vol. 192, pp. 1-13, 2017b.

[24] F. Zhang, Y.-N. Shi, J. Li, K. Wu, and H. Iwabuchi, "Variational iteration method for infrared radiative transfer in a scattering medium," Journal of the Atmospheric Sciences, vol. 74, no. 2, pp. 419-430, 2017c.

[25] B. Liebmann and C. A. Smith, "Description of a complete (interpolated) outgoing longwave radiation dataset," Bulletin of the American Meteorological Society, vol. 77, pp. 1275-1277, 1996.

[26] K. Hu, P. C. Ivanov, Z. Chen, P. Carpena, and H. E. Stanley, "Effect of trends on detrended fluctuation analysis," Physical Review E, vol. 64, no. 1, Article ID 011114, 2001. 

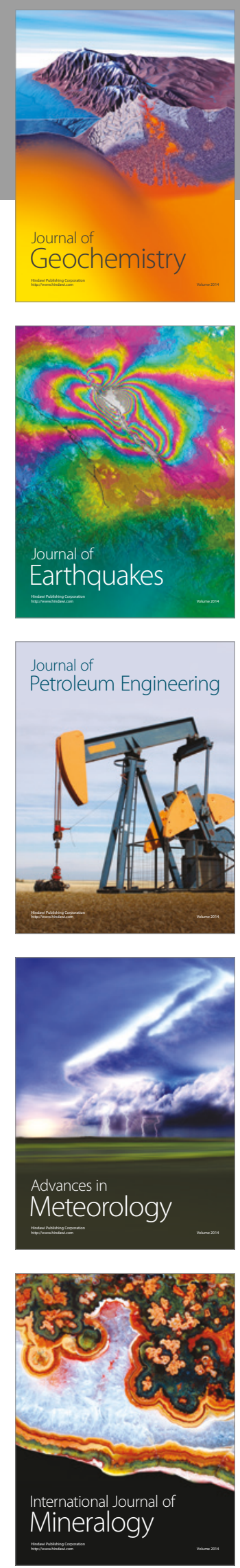
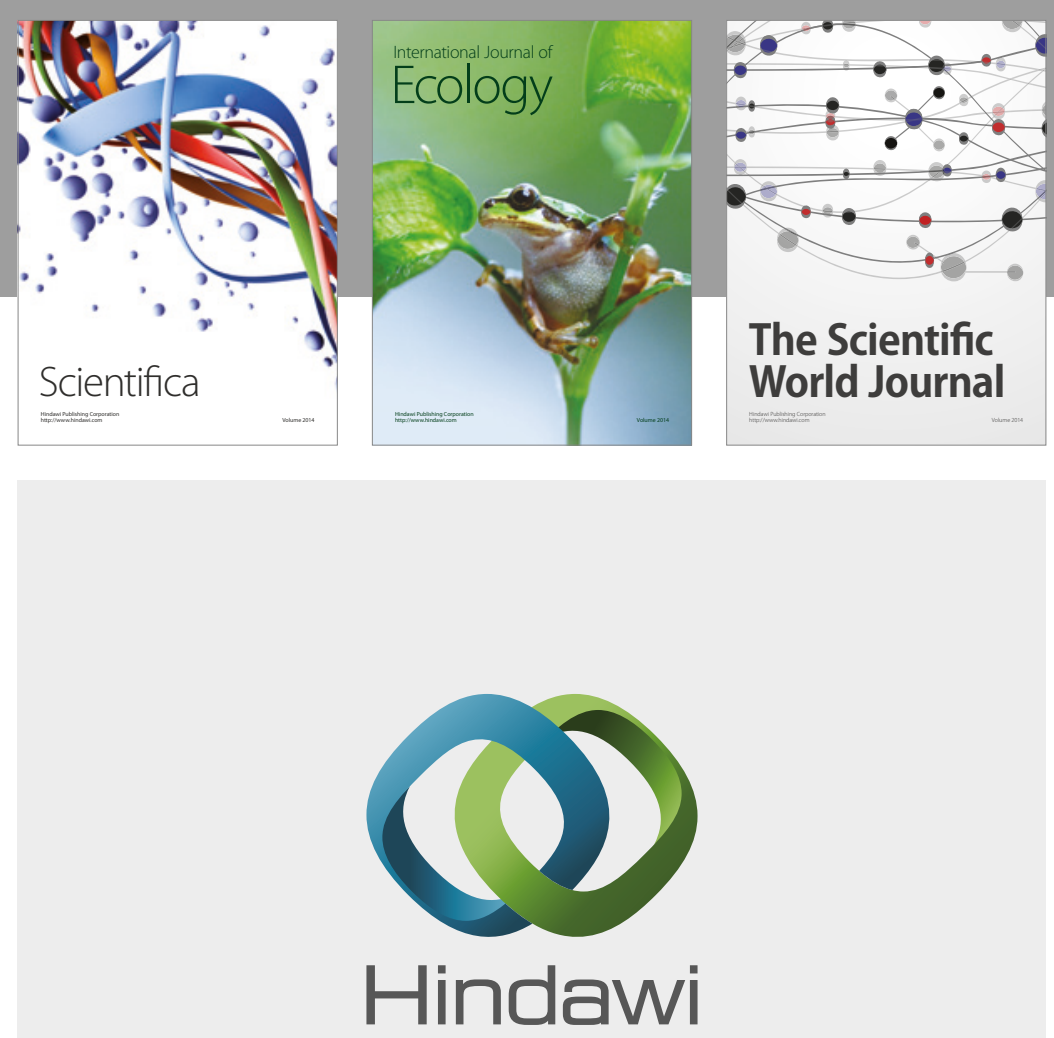

Submit your manuscripts at

https://www.hindawi.com
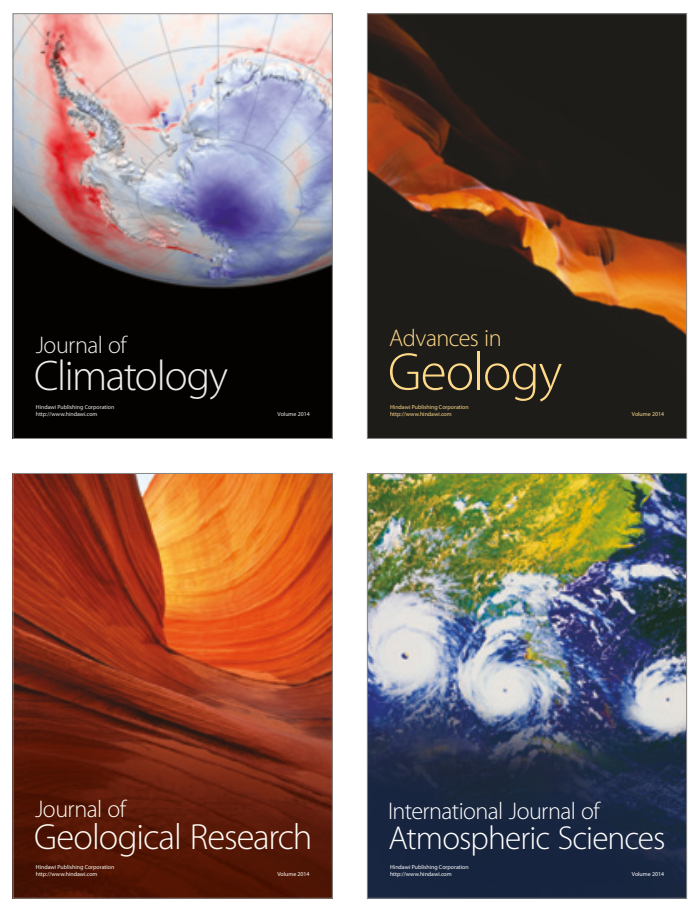

The Scientific

World Journal
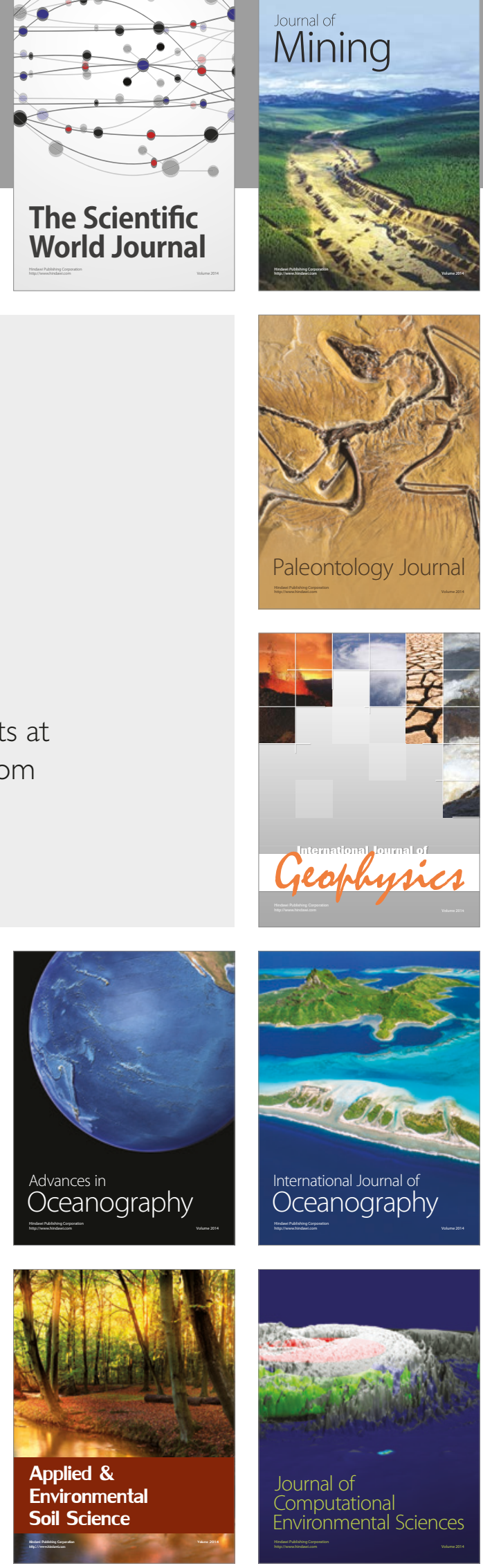